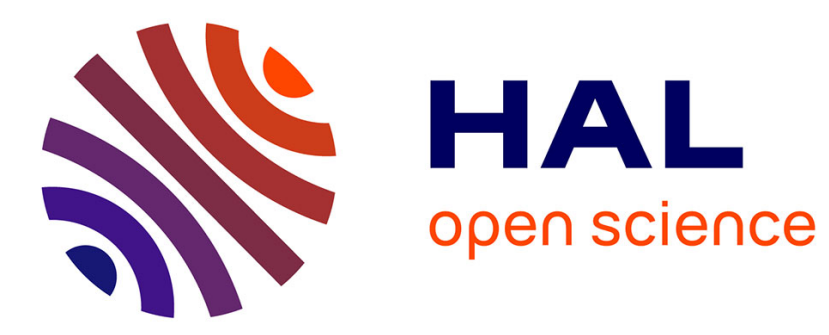

\title{
Wavelength conversion of DP-QPSK signals in a silicon polarization diversity circuit
}

Dragana Vukovic, Jochen Schroeder, Yunhong Ding, Mark D. Pelusi, Liang Bangyuan Du, Haiyan Ou, Christophe Peucheret

\section{To cite this version:}

Dragana Vukovic, Jochen Schroeder, Yunhong Ding, Mark D. Pelusi, Liang Bangyuan Du, et al.. Wavelength conversion of DP-QPSK signals in a silicon polarization diversity circuit. IEEE Photonics Technology Letters, 2015, 27 (4), pp.411-414. 10.1109/LPT.2014.2375257 . hal-01115352

\section{HAL Id: hal-01115352 https://hal.science/hal-01115352}

Submitted on 10 Feb 2015

HAL is a multi-disciplinary open access archive for the deposit and dissemination of scientific research documents, whether they are published or not. The documents may come from teaching and research institutions in France or abroad, or from public or private research centers.
L'archive ouverte pluridisciplinaire HAL, est destinée au dépôt et à la diffusion de documents scientifiques de niveau recherche, publiés ou non, émanant des établissements d'enseignement et de recherche français ou étrangers, des laboratoires publics ou privés. 


\title{
Wavelength Conversion of DP-QPSK Signals in a Silicon Polarization Diversity Circuit
}

\author{
Dragana Vukovic, Jochen Schroeder, Yunhong Ding, Mark D. Pelusi, Liang Bangyuan Du, \\ Haiyan $\mathrm{Ou}$, and Christophe Peucheret
}

\begin{abstract}
Multichannel wavelength conversion is experimentally demonstrated for high-speed $128 \mathrm{Gbit} / \mathrm{s}$ dualpolarization quadrature phase-shift keying (DP-QPSK) signals using four-wave mixing (FWM) in a polarization diversity circuit with silicon nanowires as nonlinear elements. The wavelength conversion performance is investigated for both single and threechannel input signals, showing quality factors well above $9.8 \mathrm{~dB}$ (corresponding to bit-error-ratios (BERs) better than $10^{-3}$ ) and with negligible power penalty compared to the back-to-back case.
\end{abstract}

Index Terms - Wavelength converters, photonic integrated devices, four-wave mixing, wavelength division multiplexing.

\section{INTRODUCTION}

A MONG various optical signal processing techniques, all-optical wavelength conversion appears as a key functionality to enable reconfigurability and wavelength management in future all-optical wavelength division multiplexing (WDM) networks. In such networks, multichannel wavelength converters would be required in order to minimize the number of pump lasers, decrease overall cost and energy consumption, and increase overall system flexibility.

Over the past few years, dual-polarization quadrature phaseshift keying modulation (DP-QPSK) has become the format of choice for the implementation of optical transmission systems operating at a bit rate of $100 \mathrm{Gbit} / \mathrm{s}$ and such systems are now a commercial reality. As a consequence, future wavelength conversion solutions to be deployed should be able to

Manuscript submitted July 20, 2014. This work was supported by Villum Fonden via the NATEC (NAnophotonics for Terabit Communications) Centre and the Danish Council for Independent Research (project DFF-1337-00152).

Dragana Vukovic, Yunhong Ding and Haiyan Ou are with the Department of Photonics Engineering, Technical University of Denmark, DK-2800 Kongens Lyngby, Denmark (e-mail: drvu@fotonik.dtu.dk; yudin@fotonik.dtu.dk; haou@fotonik.dtu.dk).

Jochen Schroeder and Mark Pelusi are with Centre for Ultrahigh bandwidth Devices for Optical Systems (CUDOS), School of Physics, University of Sydney, NSW 2006, Australia (e-mail: j.schroeder@physics.usyd.edu.au, pelusi@physics.usyd.edu.au).

Liang Bangyuan Du was with Department of Electrical \& Computer Systems Engineering, Monash University, Clayton, VIC 3800, Australia. He is now with Google.

Christophe Peucheret is with FOTON Laboratory, CNRS UMR 6082, ENSSAT, University of Rennes 1, 22305 Lannion, France (e-mail: christophe.peucheret@univ-rennes1.fr). accommodate this modulation format. Wavelength conversion based on four-wave mixing (FWM) is well known to be transparent to the bit rate and phase of the signal and is therefore compatible with high-speed QPSK modulation [1]. However, degenerate FWM is inherently polarization sensitive, requiring the pump to be co-polarized with the signal. In addition, in WDM networks, different channels may originate from different parts of the network and may not have the same state of polarization when reaching a common node where wavelength conversion is to be performed. Therefore, in order to be able to optically process WDM signals using FWM, a polarization insensitive scheme is required. This need is even more critical when polarization-multiplexed signals are employed.

A number of schemes based on either dual orthogonal pumps [2] or on polarization diversity loops [3] have been investigated when optical fibers are used as the nonlinear medium. However, having an integrated device is desirable since it would enable ultra-compact, on-chip, all-optical signal processing. Furthermore, integrated devices on the silicon platform offer low-cost, massive production and compatibility with mature complementary metal-oxide-semiconductor (CMOS) technology. Integrated polarization manipulation components [4], including polarization-beam splitters and rotators [5], have been the object of increased attention over the past few years, resulting in the realization of a number of functional devices [6-8]. However, a fully integrated polarization insensitive wavelength converter using an ultra-compact silicon polarization diversity (Pol-D) circuit has only been demonstrated very recently [9]. In this work, the polarization independence of the device was assessed with a single-polarization differential binary phase-shift keying (DBPSK) signal at $40 \mathrm{Gbit} / \mathrm{s}$ and interferometric detection.

In this paper, all-optical multichannel wavelength conversion of 32-Gbaud DP-QPSK signals (bit rate of $3 \times 128 \mathrm{Gbit} / \mathrm{s}$ and coherent detection) is experimentally demonstrated using FWM in a silicon-on-insulator Pol-D circuit where silicon nanowires are used as nonlinear elements. Wavelength conversion for all three WDM channels is successfully achieved with a negligible power penalty (less than $0.8 \mathrm{~dB}$ ) at an averaged quality $(\mathrm{Q})$ factor of $9.8 \mathrm{~dB}$ (equivalent to a bit-error-ratio (BER) of $10^{-3}$ ), resulting in error-free performance at a net bit rate of $3 \times 120 \mathrm{Gbit} / \mathrm{s}$ (assuming $7 \%$ forward-error-correction (FEC) overhead). To 


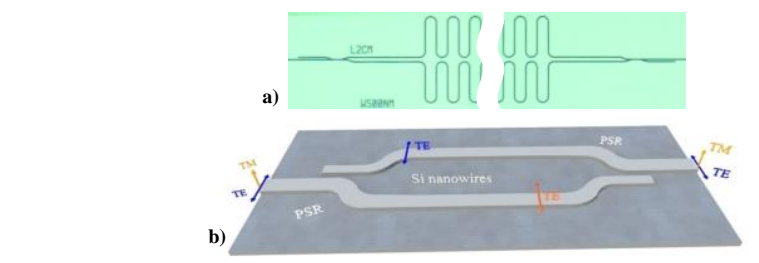

Fig. 1 (a) Microscope image and (b) schematic diagram of the polarization diversity circuit.

the best of our knowledge, this is the first demonstration of wavelength conversion for dual polarization signals using a silicon nanowire as nonlinear element.

\section{POLARIZATION DIVERSITY CIRCUIT}

The Pol-D circuit fabricated on a silicon-on-insulator (SOI) wafer consists of two identical polarization splitters and rotators (PSRs) connected by two identical $2-\mathrm{cm}$ long silicon nanowires, with cross sections of $250 \mathrm{~nm} \times 500 \mathrm{~nm}$. The input PSR is based on a tapered waveguide $\mathrm{TM}_{0}$-to-TE $\mathrm{TE}_{1}$ mode converter [10] followed by a tapered directional coupler (DC)based $\mathrm{TE}_{0} \& \mathrm{TE}_{1}$ mode splitter [11]. In case of $\mathrm{TE}_{0}$ input, light propagates through the adiabatic taper without any change, while in case of $\mathrm{TM}_{0}$ input, light is converted to the $\mathrm{TE}_{1}$ mode during the adiabatic tapering [10]. The mode splitter then separates the $\mathrm{TE}_{0}$ and $\mathrm{TE}_{1}$ modes by coupling them into two output waveguides on the $\mathrm{TE}_{0}$ mode. The two $\mathrm{TE}_{0}$ signal beams propagate separately through the silicon nanowires and interact nonlinearly with the pump. At the waveguides outputs, an identical PSR combines back the two $\mathrm{TE}_{0}$ beams so that the entire device shows polarization insensitive transmission. In particular, provided the same fraction of pump power is coupled to the $\mathrm{TE}_{0}$ and $\mathrm{TM}_{0}$ modes at the input of the circuit, the same FWM conversion efficiencies will be obtained in each of the silicon nanowires, resulting in polarization-insensitive wavelength conversion. Furthermore, since both waveguides, which represent the main propagation length in the device, operate on the same (TE) polarization, the differential group delay of the component is expected to be negligible. Both input and output of the device are equipped with silicon inverse tapers in order to reduce coupling loss to tapered fibers to $1.5 \mathrm{~dB} /$ facet. The minimum insertion loss of the device was around $13 \mathrm{~dB}$ with about $2.6 \mathrm{~dB}$ residual polarization-dependent loss (PDL). Typical propagation loss of the $\mathrm{Si}$ nanowires is $4.2 \mathrm{~dB} / \mathrm{cm}$. A microscope image and schematic structure of the device are shown in Fig. 1(a) and (b). More details on the design and fabrication of the device can be found in $[9,10]$.

\section{EXPERIMENTAL SETUP}

Fig. 2 shows the experimental setup for all-optical wavelength conversion. In order to generate $3 \times 32$ Gbaud DP-QPSK data signals, three continuous-wave $(\mathrm{CW})$ distributed feedback (DFB) lasers with $100-\mathrm{GHz}$ channel spacing at $1548.3 \mathrm{~nm}$, $1549.1 \mathrm{~nm}$ and $1549.9 \mathrm{~nm}$, respectively, were coupled together and modulated in an in-phase/quadrature (IQ) modulator driven by two pseudo-random binary sequences

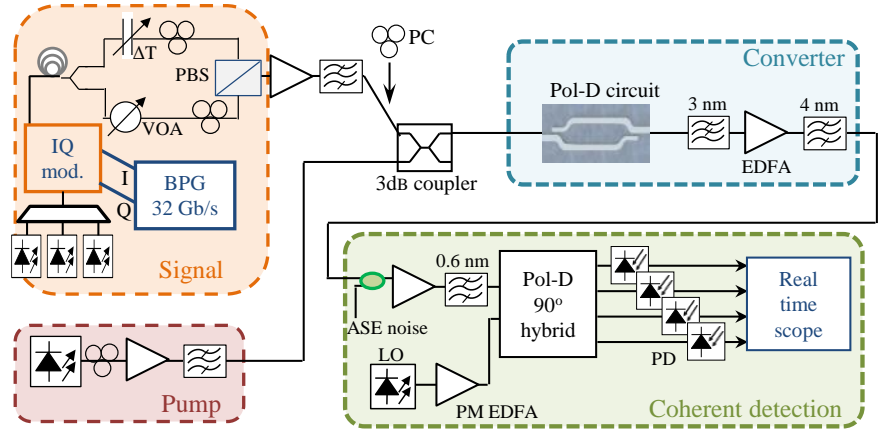

Fig. 2. Experimental setup for wavelength conversion of a $3 \times 32$ Gbaud DPQPSK signal

(PRBSs) of lengths $2^{20}-1$ and $2^{15}-1$ generated from a bitpattern generator (BPG). The three WDM channels were then decorrelated by propagation over $2.8 \mathrm{~km}$ of standard single mode fiber. Polarization-multiplexing (Pol-Mux) was emulated by splitting the QPSK signals into two branches, delaying one QPSK stream with respect to the other by about $1 \mathrm{~ns}$, and then recombining them with orthogonal polarizations in a polarization beam splitter (PBS). The signals were amplified in an erbium-doped fiber amplifier (EDFA) followed by a 3-nm optical band-pass filter (OBPF) and combined with a pump in a $3-\mathrm{dB}$ coupler. A polarization controller in the signal arm was used to check the polarization insensitivity of the device. In the case of single channel wavelength conversion, the signal was generated from an external cavity laser (ECL) at $1549.7 \mathrm{~nm}$. The pump was generated from an ECL at $1552.8 \mathrm{~nm}(1551.3 \mathrm{~nm}$ in the single channel case), amplified by an EDFA and finally filtered by a 1-nm OBPF. The polarization of the pump at the input of the device was controlled by a polarization controller. Due to the presence of $2.6 \mathrm{~dB}$ of PDL in our sample, which is mainly due to different coupling loss to the chip for the TE and TM modes, the pump polarization was aligned so that its power in the TE and TM modes was the same at the input of the PSR. Since the PSR itself has negligible PDL, having equal pump power levels in the TE and TM modes at its output will lead to polarization-independent wavelength conversion, hence identical conversion efficiencies for the two polarization tributaries of the DP-QPSK signals. At the output of the Pol-D circuit, two OBPFs were used to select the converted channels. The filtered idler was amplified and input to a polarizationdiversity coherent receiver for detection and Q measurements. The coherent receiver consists of an optical 90-degree hybrid circuit, four balanced photodetectors (PDs) and a tunable ECL as local oscillator (LO). The outputs of the PDs were then input to a 4-channel real-time oscilloscope (Agilent DSOX96204Q) with 80 Gsamples/s sampling rate and $32 \mathrm{GHz}$ electrical bandwidth. The captured data were processed offline through standard steps of digital signal processing (DSP), which include data resampling, polarization recovery (constant modulus algorithm), and phase recovery (Viterbi-Viterbi algorithm). The performance was characterized by evaluating the $\mathrm{Q}$ factor from the Cartesian spreads of the constellations, as presented in [12], over 200000 collected symbols in the case of multichannel wavelength conversion and 100000 collected symbols for single channel wavelength conversion. 


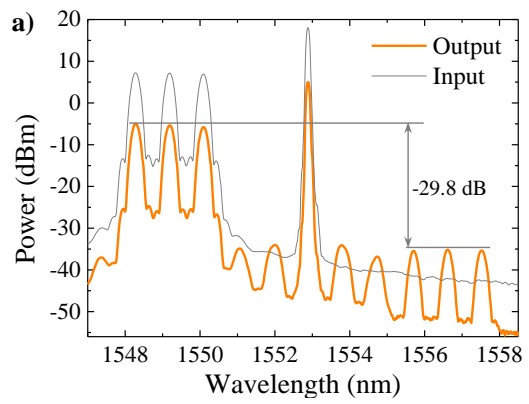

Fig. 3 Spectra at the input and output of the polarization diversity circuit $(0.1 \mathrm{~nm}$ resolution) for (a) multichannel and (b) single channel wavelength conversion of DP-QPSK signals at $128 \mathrm{Gbit} / \mathrm{s}$.

\section{RESULTS AND DISCUSSION}

The spectra measured at the output of the device for multichannel and single channel wavelength conversion are shown in Fig. 3. The total signal and pump power levels at the input of the Pol-D circuit in the multichannel case were $18.3 \mathrm{dBm}$ and $21 \mathrm{dBm}$, respectively, leading to a conversion efficiency (CE, defined as the ratio between the idler power and signal power at the output of the device) of $-29.8 \mathrm{~dB}$. In the case of single channel wavelength conversion the signal and pump power levels were $15.2 \mathrm{dBm}$ and $18.7 \mathrm{dBm}$, respectively, leading to $-27.4 \mathrm{~dB}$ of conversion efficiency. The different conversion efficiencies for single channel and multichannel operation could be due to the nonlinear absorption mechanism. Even though higher CEs could be obtained by increasing the total pump power further, the scheme is limited fundamentally by free-carrier absorption induced by two-photon absorption, unless more complex waveguide structures with transverse p-i-n junctions are implemented [13], and practically by the damage threshold of the chip input facet.

Fig. 4 shows the averaged Q-factor performance of the converted signal as a function of the optical signal-to-noise ratio (OSNR, defined in a $0.1 \mathrm{~nm}$ noise bandwidth) at the input of the receiver. In these measurements, the output power of the EDFA after the Pol-D circuit was optimized so that the OSNR of the converted signals was maximized at the input of the coherent receiver. The output power of the EDFA before the coherent receiver was set to the maximum power that could be input to the receiver. The OSNR of the converted signals was then degraded using a broadband amplified spontaneous emission (ASE) noise source before the coherent receiver, as indicated in Fig. 2. The back-to-back (B2B) performance was measured directly at the output of the DPQPSK transmitter, and compared with the performance of the converted signal. The Q-factor was averaged over all three channels and both polarizations for each channel. A small power penalty, lower than $0.5 \mathrm{~dB}$ at a $\mathrm{Q}$ of $9.8 \mathrm{~dB}$, corresponding to a BER of $10^{-3}$, was measured for the converted signals in case of WDM as well as single channel conversion, as shown in Fig. 4(a) and (b), respectively. In order to investigate the origin of the penalty, the performance of a converted signal was characterized when only one signal polarization was present at the device input (64-Gbit/s QPSK signal). The performance is shown in Fig. 4 (b). In this case,
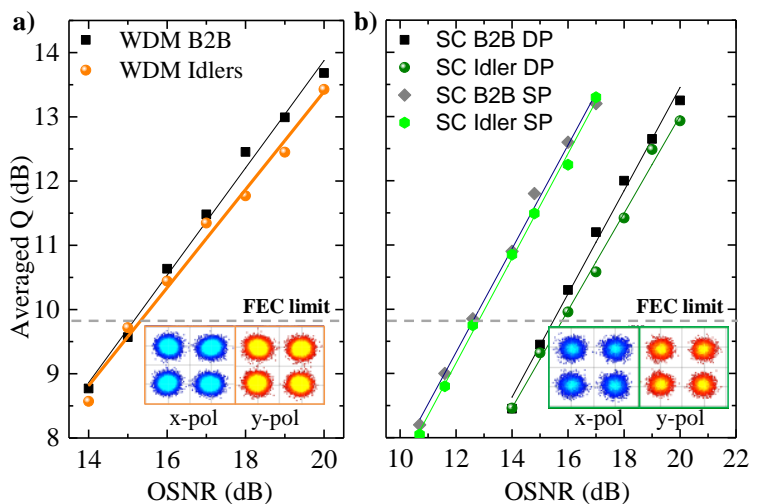

Fig. 4. Comparison between averaged $Q$-factor as a function of OSNR for (a) three WDM channel and (b) single channel wavelength conversion. The insets are showing constellation diagrams for converted signals of (a) the worst WDM channel, (b) single channel for $20 \mathrm{~dB}$ of OSNR. (SC - single channel, $\mathrm{SP}$ - single polarization)

wavelength conversion was achieved without any power penalty, indicating that the main source of residual penalty in the DP case is caused by imperfections in the PSR, leading to some residual cross-talk between the two polarization tributaries. Nevertheless, a power penalty of less than $0.5 \mathrm{~dB}$ in the dual-polarization case clearly remains within tolerable range.

Fig. 5 shows the OSNR penalty with respect to back-toback at a fixed $\mathrm{Q}$ of $9.8 \mathrm{~dB}$ for each polarization tributary of all three converted channels, as well as for wavelength conversion of a single channel. The difference in performance between the two polarization tributaries can also be attributed to imperfections in the PSRs. However, this difference (less than $0.4 \mathrm{~dB}$ ) is fairly small. As it can be seen from the figure, there is no significant excess OSNR penalty when converting more than one channel. By optimizing the pump power as well as the signal power it would be possible to convert even more WDM channels. The main limitation for the present implementation would be the conversion bandwidth of the waveguides used as nonlinear elements, which is about $18 \mathrm{~nm}$, as shown in Fig. 6. The FWM conversion efficiencies have been characterized as a function of the pump-signal wavelength detuning using a $\mathrm{CW}$ pump for $\mathrm{CW}$ input signals polarized along two orthogonal directions. Identical conversion bandwidths and maximum conversion efficiencies were obtained, which confirms the expected operation of the polarization diversity circuit. The limited conversion bandwidth is not an intrinsic limitation of the scheme, and is due to the dispersion of the nonlinear waveguides, which was estimated using a vectorial finite-difference mode solver [13] to be $684 \mathrm{ps} / \mathrm{nm} / \mathrm{km}$ at $1550 \mathrm{~nm}$. Larger conversion bandwidths could be obtained through dispersion engineering by optimizing the cross-sections of the nonlinear waveguides. 


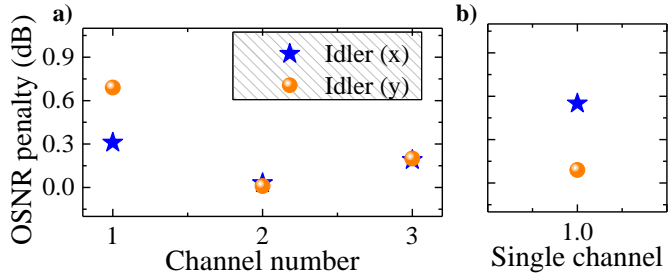

Fig. 5. OSNR penalty with respect to B2B at a fixed BER of $10^{-3}$ for both polarization tributaries in (a) three WDM channel conversion and (b) single channel conversion.

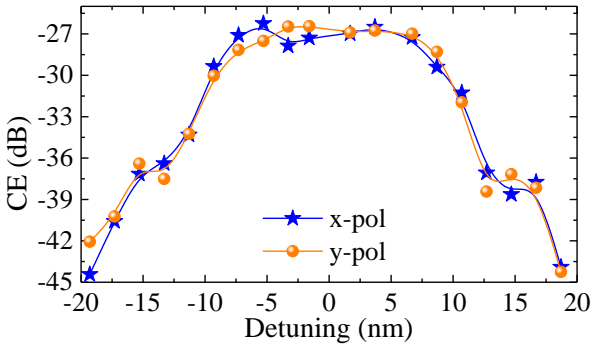

Fig. 6. CW characterization of the FWM conversion bandwidth of the Pol-D circuit. $\mathrm{x}$ and $\mathrm{y}$ are two orthogonally polarized $\mathrm{CW}$ waves.

\section{CONCLUSION}

Using a polarization diversity circuit with nonlinear waveguides fully integrated on the silicon platform, we have successfully demonstrated wavelength conversion of a $3 \times 128 \mathrm{Gbit} / \mathrm{s}$ DP-QPSK signal based on FWM. The performance of the converted signals has been characterized through Q measurements, showing excellent performances for all converted channels. This is, to the best of our knowledge, the first successful demonstration of wavelength conversion of dual polarization QPSK signals on the silicon platform.

\section{REFERENCES}

[1] J. Yu and M. F. Huang, "Wavelength conversion for 112Gbit/s PolMuxRZ-QPSK signals based on four-wave mixing in high-nonlinear fiber using digital coherent detection," in Proc. Eur. Conf. Opt. Commun., 2008, Mo.3.C.5
[2] K. Inoue, "Polarization independent wavelength conversion using fiber four-wave mixing with two orthogonal pump lights of different frequencies," J. Lightwave Technol. vol. 12, pp. 1916-1920, 1994.

[3] T. Hasegawa, K. Inoue, and K. Oda, "Polarization independent frequency conversion by fiber four-wave mixing with a polarization diversity technique," IEEE Photon Technol. Lett. vol. 5, pp. 947-949, 1993.

[4] D. Dai, L. Liu, S. Gao, D.-X. Xu, and S. He, "Polarization management for silicon photonic integrated circuits," Laser Photonics Rev. vol. 7, pp. 303-328, 2013

[5] M. R. Watts, M. Qi, T. Barwicz, L. Socci, P. T. Rakich, E. P. Ippen, H. I. Smith, H. A. Haus, "Towards integrated polarization diversity: design, fabrication, and characterization of integrated polarization splitters and rotators," in Proc. Opt. Fiber Conf., 2005, PDP11.

[6] H. Fukuda, K. Yamada, T. Tsuchizawa, T. Watanabe, H. Shinojima, and S. Itabashi, "Silicon photonic circuit with polarization diversity," Opt. Express vol. 16, pp. 4872-4880, 2008.

[7] T. Barwicz, M. Watts, M. Popovic, P. Rakich, L. Socci, F. Kartner, E. Ippen, and H. Smith," Polarization-transparent microphotonic devices in the strong confinement limit," Nature Photon., vol. 1, pp. 57, 2007.

[8] W. Bogaerts, D. K. Taillaert, P. Dumon, D. Van Thourhout, and R. Baets, "A polarization-diversity wavelength duplexer circuit in siliconon-insulator photonic wires," Opt. Express., vol. 15, pp. 1567, 2007.

[9] D. Vukovic, Y. Ding, H. Hu, H. Ou, L. K. Oxenløwe, and C. Peucheret, "Polarization-insensitive wavelength conversion of $40 \mathrm{~Gb} / \mathrm{s}$ NRZ-DPSK signals in a silicon polarization diversity circuit," Opt. Express, vol. 22, pp. 12467-12474, 2014.

[10] Y. Ding, H. Ou, and C. Peucheret, "Wideband polarization splitter and rotator with large fabrication tolerance and simple fabrication process," Opt. Letters, vol. 38, pp. 1227-1229, 2013.

[11] Y. Ding, J. Xu, F. D. Ros, B. Huang, H. Ou, and C. Peucheret, "On-chip two-mode division multiplexing using tapered directional coupler-based mode multiplexer and demultiplexer," Opt. Express, vol. 21, pp. 10376$10382,2013$.

[12] A. J. Lowery, L. B. Du, and J. Armstrong "Performance of optical OFDM in ultralong-haul WDM lightwave systems" $J$. of Lightwave Technol., vol. 25, pp. 131-138, 2007.

[13] A. Gajda, L. Zimmermann, M. Jazayerifar, G. Winzer, H. Tian, R. Elschner, T. Richter, C. Schubert, B. Tillack, and K. Petermann, "Highly efficient $\mathrm{CW}$ parametric conversion at $1550 \mathrm{~nm}$ in SOI waveguides by reverse biased p-i-n junction," Opt. Express, vol. 20, pp. 13100-13107, 2012.

[14] A. B. Fallahkhair, K. S. Li, and T. E. Murphy, "Vector finite difference modesolver for anisotropic dielectric waveguides," J. Lightwave Technol., vol. 26, pp. 1423-1431, 2008 